\title{
RESPONSABILIDAD DE LA EMPRESA PRINCIPAL EN PREVENCIÓN DE RIESGOS LABORALES: ÁMBITO DE APLICACIÓN Y SIGNIFICADO DEL REQUISITO CONTRATAS Y SUBCONTRATAS PROPIAS DEL GIRO DE LA EMPRESA PRINCIPAL
}

\author{
Raúl Fernández Toledo* \\ Universidad de Chile
}

\begin{abstract}
RESUMEN: El propósito de este trabajo es determinar los requisitos que son necesarios para que la empresa principal tenga obligaciones y responsabilidad en la prevención de riesgos laborales de los trabajadores contratistas y subcontratistas, poniendo especial énfasis en el requisito que la contrata y subcontrata tenga por objeto una obra, faena o servicio propio del giro de la empresa principal exigido por el artículo 66 bis de la Ley $N^{\circ} 16.744$ sobre Accidentes del Trabajo y Enfermedades Profesionales. Para ello se expondrán las distintas posiciones existentes en la doctrina y jurisprudencia nacional sobre este último requisito como también las consecuencias jurídicas que derivan de su existencia o inexistencia.
\end{abstract}

Palabras clave: empresa principal - giro propio - lugar de trabajo - prevención de riesgos - contratista subcontratista

\begin{abstract}
The purpose of this work is to determine the requirements necessary for the main company to have obligations and liability in the prevention of labor risks of its contractor's and subcontractor's employees, with special emphasis in the requirement that the contract and subcontract is for the execution of a work or service that is within the corporate purpose of the main company, requirement set forth in article 66 bis of Law $N^{\circ} 16.744$ of Labor Accidents and Professional Diseases. For such purpose, the distinct positions existent in Chilean doctrine and jurisprudence with respect to this last requirement will be described, as well as the legal consequences arising from its existence or inexistence.
\end{abstract}

Key words: main company-place of work-risk prevention-contractor-subcontractor

\section{INTRODUCCIÓN}

La subcontratación no solo es una forma de organización productiva descentralizada, que permite a las empresas desarrollar de mejor forma sus actividades productivas, también es un factor de siniestralidad laboral, al llevar aparejado un cúmulo de riesgos que actúan sobre los trabajadores de los contratistas y subcontratistas, que determina su mayor susceptibilidad a los riesgos laborales ${ }^{1}$.

\footnotetext{
Abogado. Licenciado en Ciencias Jurídicas y Sociales, Universidad de Concepción. Magíster en Derecho del Trabajo y de la Seguridad Social, Universidad de Talca-Universidad de Valencia. Instructor, Departamento de Derecho del Trabajo y de la Seguridad Social, Facultad de Derecho, Universidad de Chile. Contacto: rfernandez@ derecho.uchile.cl

1 Entre estos factores de riesgos se puede señalar principalmente: a) La concurrencia de actividades empresariales en un mismo lugar de trabajo que implica que los trabajadores queden expuestos no solo a los riesgos originados por su propio empleador sino también a los causados por las restantes empresas; b) desconocimiento del medio de trabajo por los trabajadores de las empresas contratistas y subcontratistas que desarrollan su actividad laboral en un lugar de trabajo ajeno; c) carácter temporal que suelen tener los contratos de trabajo de los trabajadores que intervienen en estas estructuras empresariales complejas; d) despreocupación de la empresa principal respecto de los trabajadores de la contratista o subcontratista a quienes considera
} 
Raúl Fernández Toledo / Responsabilidad de la empresa principal en prevención de riesgos laborales: ámbito de aplicación y significado del requisito contratas y subcontratas propias del giro de la empresa principal

Debido a esta circunstancia el legislador laboral se ha hecho cargo de la relación existente entre la descentralización productiva - constituida por la subcontratación - y la siniestralidad laboral, la que ha sido puesta de manifiesto en la actual regulación normativa del trabajo en régimen de subcontratación, estableciendo, primero, numerosas obligaciones y deberes compartidos de prevención de riesgos laborales entre las empresas de la cadena productiva y,luego, las responsabilidades que pueden derivarse del incumplimiento de estas obligaciones y deberes. Normativa que pone especial énfasis en la responsabilidad que le corresponde a la empresa principal respecto de los trabajadores de las empresas contratistas y subcontratistas que le prestan servicios, en lo tocante a la prevención de los riesgos laborales. Sin embargo, esta responsabilidad no es absoluta, debido a que la subcontratación debe cumplir ciertas exigencias para que nazcan a la vida jurídica las obligaciones preventivas de riesgos laborales que le corresponden a la empresa principal.

En nuestra opinión la normativa laboral nacional restringe el campo de aplicación de la obligación de seguridad y el régimen de responsabilidad de la empresa principal en la prevención de los riesgos laborales de los trabajadores contratistas y subcontratistas, no haciéndola aplicable a todo supuesto de subcontratación. El tenor literal de las diversas disposiciones legales da cuenta de ello. Definido qué es el trabajo en régimen de subcontratación por el artículo 183 - A del Código del Trabajo, el artículo 183 - E, que es la norma de cierre del párrafo sobre el trabajo en régimen de subcontratación, establece que:

\begin{abstract}
"Sin perjuicio de las obligaciones de la empresa principal, contratista y subcontratista respecto de sus propios trabajadores en virtud de lo dispuesto en el artículo 184, la empresa principal deberá adoptar las medidas necesarias para proteger eficazmente la vida y salud de todos los trabajadores que laboran en su obra, empresa o faena, cualquiera sea su dependencia, en conformidad a lo dispuesto en el artículo 66 bis de la Ley $\mathrm{N}^{\circ} 16.744$ y el artículo $3^{\circ}$ del decreto supremo $\mathrm{N}^{\circ} 594$, de 1999, del Ministerio de Salud".
\end{abstract}

Disposición, que según se puede observar, se remite a una norma legal y a otra reglamentaria elevada a rango legal ${ }^{2}$ para establecer cuándo la empresa principal debe cumplir las obligaciones de prevención de riesgos laborales respecto de los trabajadores contratistas y subcontratistas y bajo qué condiciones ${ }^{3}$.

ajenos. Véase: Mrñarro YaninI, María. "La prevención de riesgos laborales en las contratas y subcontratas", en: Documentación Laboral, $\mathrm{N}^{\circ}$ 68, Vol. II, pp. 84 - 87. Madrid, España, 2003

2 Lizama Portal, Luis y Ugarte Cataldo, José Luis, Subcontratación y Suministro de Trabajadores. Santiago, Chile: LexisNexis, 2007, p. 50; Diez Schwerter, José Luis, "Responsabilidad civil derivada de accidentes del trabajo y enfermedades profesionales en Chile: evolución, funcionamiento y propuestas de racionalización”, en: Revista de Derecho, $\mathrm{N}^{\circ} 225$ - 226, pp. 250 Concepción, Chile: Facultad de Derecho, Universidad de Concepción, 2009 Núñez GonzÁtez, Cayetano. Prevención de riesgos laborales en Chile. Alcance y contenido del artículo 184 del Código del Trabajo. Santiago, Chile: Librotecnia, 2013, p. 168.

3 Véase sobre la discusión del alcance de la obligación de seguridad de la empresa principal prevista en el artículo $183 \mathrm{E}$ del Código del Trabajo: Núñez González, Cayetano, op. cit. (n. 2), pp. 170 - 173. Es discutible cuáles son las obligaciones en prevención de riesgos laborales que la empresa principal tiene sobre los trabajadores contratistas y subcontratistas. Controversia que deriva del distinto sentido y alcance que puede otorgársele al artículo $183 \mathrm{E}$ del Código del Trabajo. Una posibilidad es entender que la empresa principal tiene la obligación de seguridad en los mismos términos que el empleador directo, además, de la obligación de vigilancia y coordinación prevista en el mismo artículo 66 bis de la Ley $\mathrm{N}^{\circ} 16.744$ y la obligación establecida en el artículo 3 del Decreto Supremo 594 del Ministerio de Salud. Otra alternativa, es sostener que las únicas obligaciones en prevención de riesgos laborales que tiene la empresa principal son las reguladas en el artículo 3 del Decreto Supremo 594 del Ministerio de Salud y en el artículo 66 bis de la Ley $\mathrm{N}^{\circ} 16.744$, constituyendo el artículo $183 \mathrm{E}$ del Código del Trabajo solamente una norma de remisión. La doctrina mayoritaria si inclina por la primera tesis, así, entre otros: NúÑEZ GoNZÁLEZ, Cayetano, op. 


\section{El artículo 66 bis de la Ley $\mathrm{N}^{\circ} 16.744$ establece que:}

"Los empleadores que contraten o subcontraten con otros la realización de una obra, faena o servicios propios de su giro, deberán vigilar el cumplimiento por parte de dichos contratistas o subcontratistas de la normativa relativa a higiene y seguridad, debiendo para ello implementar un sistema de gestión de la seguridad y salud en el trabajo para todos los trabajadores involucrados, cualquiera que sea su dependencia, cuando en su conjunto agrupen a más de 50 trabajadores".

Especificando en los incisos siguientes de la misma disposición en qué consiste dicho sistema, el que es desarrollado en el Decreto Supremo 76 del Ministerio del Trabajo y Previsión Social del año 2006. Por su parte, el artículo $3^{\circ}$ del Decreto Supremo 594 de 1999 del Ministerio de Salud, dispone "la empresa está obligada a mantener en los lugares de trabajo las condiciones sanitarias y ambientales necesarias para proteger la vida y salud de los trabajadores que en ellos se desempeñen, sean éstos dependientes directos suyos o lo sean de terceros contratistas que realizan actividades para ella".

Las normas antes transcritas no sólo establecen las obligaciones que corresponden a la empresa principal en prevención de riesgos laborales respecto de los trabajadores dependientes de los contratistas y subcontratistas que le prestan servicios, sino también indican cuáles son los requisitos necesarios para el surgimiento de aquellas obligaciones. Cuestión esta última que no es intrascendente, porque si no concurren los mismos, no tiene la empresa principal responsabilidad en prevención de riesgos respecto de los trabajadores contratistas y subcontratistas o, al menos, el número de obligaciones en prevención de riesgos laborales es menor. De ahí, que sea necesario establecer como primera cuestión cuáles son esos requisitos. Los cuáles no han sido establecidos con claridad por la doctrina nacional, la que se ha dedicado a estudiar, principalmente, la responsabilidad civil de la empresa principal ${ }^{4}$, el contenido y tipo de obligaciones en prevención de riesgos laborales de la empresa principal $^{5}$.

cit. (n. 2), pp. 170 - 173; Gajardo Harboe, María Cristina. "Obligaciones en materia de higiene y seguridad en el trabajo: los cambios de la Ley de Subcontratación”, en: Revista Laboral Chilena, año 19, No 176, pp. 82 - 90 . Santiago, Chile, 2009. En cambio, la doctrina minoritaria se inclina por la segunda posición, destacando Enrique Pérez, quien luego de transcribir el inciso $1^{\circ} \mathrm{del}$ artículo $183 \mathrm{E}$ del Código del Trabajo, sostiene: "De este deber especial de protección que el legislador impone a la empresa principal en materia de protección de la seguridad y salud de los trabajadores en régimen de subcontratación, se distinguen dos obligaciones legales: la primera de ellas, es el cumplimiento de la disposición del artículo $3^{\circ}$ del D.S. $\mathrm{N}^{\circ} 594$, de 1999 , del Ministerio de Salud y la segunda, el cumplimiento de las disposiciones establecidas en el artículo 66 bis de la ley 16.744, en orden a establecer un Sistema de Gestión de Seguridad y Salud en el Trabajo, constituir y poner en funcionamiento un comité paritario de higiene y seguridad de faena, y constituir y poner en funcionamiento un departamento de prevención de riesgos laborales en faena", PÉrez Mendoza, Enrique. Normas de seguridad y salud en el trabajo establecidas en la Ley $N^{\circ} 20.123$ y en el Reglamento $N^{\circ} 76 / 2007$ del Ministerio del Trabajo y Previsión Social. Documento inédito no publicado, pp. 3 - 4.

4 Véase, entre otros: Prado López, Pamela. “Crítica a la configuración de la responsabilidad civil de la empresa principal y de la empresa usuaria en la Ley N ${ }^{\circ} 20.123$ ”. En: V.V.A.A. Estudios de Derecho Civil III. Jornadas Nacionales de Derecho Civil Valparaiso 2007. Santiago, Chile: LegalPublishing, 2008, pp. 857 - 872; Diez SCHWErTer, José Luis. "Responsabilidad civil derivada de accidentes del trabajo y enfermedades profesionales. Aspectos relevantes de su regulación y operatoria actual", en: Revista de Derecho, $N^{\circ}$ XXXI. pp. 169 - 170; Valparaíso, Chile: Escuela de Derecho, Pontificia Universidad Católica de Valparaíso. 2008, BARRientos Zamorano, Marcelo, "La obligación de seguridad en la subcontratación laboral: previsibilidad del hecho y del daño", en: Revista Chilena de Derecho, Vol. 39, $\mathrm{N}^{\circ}$ 1, pp. 87 - 111 Santiago, Chile: Facultad de Derecho, Pontificia Universidad Católica de Chile, 2012, .

5 Véase, entre otros: Gajardo Harboe, María Cristina, op. cit. (n. 3), pp. 81 - 95; Barrientos Zamorano, Marcelo, op. cit. (n. 4), pp. 77 - 86; NúÑ̃z GoNZÁLeZ, Cayetano, op. cit. (n. 2), pp. 161 - 180. 
Raúl Fernández Toledo / Responsabilidad de la empresa principal en prevención de riesgos laborales: ámbito de aplicación y significado del requisito contratas y subcontratas propias del giro de la empresa principal

En primer lugar, es necesario que exista un supuesto de trabajo en régimen de subcontratación $^{6}$, que cumpla en forma copulativa con los requisitos de la definición contenida en el artículo 183 A del Código del Trabajo, únicos que hacen procedentes dicha forma de descentralización productiva, requiriéndose, necesariamente, para su surgimiento de una empresa principal ${ }^{7}$. El tenor literal de los artículos $183 \mathrm{E}$ del Código del Trabajo, 66 bis de la Ley $\mathrm{N}^{\circ} 16.744$ y 3 del Decreto Supremo $\mathrm{N}^{\circ} 594$ del Ministerio de Salud ponen de manifiesto este requisito al emplear los términos "empresa principal", "contratistas", subcontratistas", "contratas", "subcontratas" y "terceros contratistas", expresiones propias del fenómeno de la descentralización productiva denominado trabajo en régimen de subcontratación. Lo que es reforzado con la terminología empleada por el Decreto Supremo 76 del Ministerio del Trabajo y Previsión Social, que alude reiteradamente a la subcontratación.

En segundo lugar, se requiere que la contrata y/o subcontrata se desarrolle en el mismo "lugar de trabajo" que la actividad realizada por la empresa principal. Requisito que emana del inciso $3^{\circ}$ del artículo 66 bis de la Ley $N^{\circ} 16.744$ y del artículo $3^{\circ}$ del Decreto Supremo $N^{\circ} 594$ del Ministerio de Salud, preceptos a los que se remite el artículo $183 \mathrm{E}$ del Código del Trabajo, constituyendo una condición necesaria para el nacimiento de todas las obligaciones sobre prevención de riesgos laborales de la empresa principal. Lo que se explica, porque solamente teniendo la supervisión del lugar de trabajo la empresa principal podrá adoptar las medidas necesarias para cumplir sus obligaciones de protección, de lo contrario, al no tener el control del espacio físico de producción, le es imposible velar por la seguridad y salud en el trabajo de los dependientes que se desempeñan en régimen de subcontratación ${ }^{8}$. Por lo mismo, sino concurre el requisito del lugar de trabajo en la subcontratación no le es exigible ninguna obligación de prevención de riesgos laborales en calidad de empresa principal y, por tanto, no será responsable de los accidentes del trabajo que sufran los trabajadores contratistas 9 .

\footnotetext{
6 NúÑez González, Cayetano, op. cit. (n. 2), p. 169.

7 Sentencia del Juzgado de Letras, Garantía y Familia de Pichilemu, 10 de marzo de 2014, causa RIT I - 21 - 2013 exige solamente este requisito para que nazca a la vida del derecho la obligación de seguridad prevista en el artículo $183 \mathrm{E}$ del Código del Trabajo. En Cfr. http://www. laboral.poderjudicial.cl/SITLAPORWEB/InicioAplicacionPortal.do [consultada el 10 de noviembre de 2014].
}

8 Debe tenerse presente que es discutido en la doctrina nacional si es requisito de existencia de la subcontratación que los servicios subcontratados deban ejecutarse en un lugar de trabajo controlado por la empresa principal. La discusión emana del requisito que consiste en que los servicios u obras contratadas deben ejecutarse o realizarse "en" la obra, empresa o faena de la empresa principal, establecido en el artículo 183 - A del Código del Trabajo. Una primera línea interpretativa sostiene que por el uso que hace la ley de la preposición "en" en la definición legal de subcontratación, requiere ésta para su configuración y nacimiento a la vida del derecho, de la concurrencia de una circunstancia geográfica, locativa o espacial. Esta circunstancia consiste en que los servicios deben desarrollarse o las obras deberán ejecutarse, en un lugar cuyo dominio, posesión o mera tenencia corresponde a la empresa principal. Si los servicios se desarrollan o la obra contratada se ejecuta fuera de dicho espacio, no existirá subcontratación jurídica laboral en los términos protectivos que regula el Código del Trabajo (PALAVECINo CÁCERES, Claudio. Subcontratación. Régimen jurídico del trabajo subcontratado y del suministro de personal. Santiago, Chile: Editorial Jurídica, 2006, pp. 36 - 38). La segunda tesis, en cambio, sostiene que el elemento locativo no es determinante, y que lo definitorio es que el contratista o subcontratista participede algún proceso productivo de la empresa principal, independientemente del lugar donde se prestarán los servicios, por tanto, existirá subcontratación, tanto si las obras o servicios que ejecutan los trabajadores del contratista se desarrollan en las instalaciones o espacios físicos propios de la persona natural o jurídica dueña de la obra, empresa o faena, como fuera de éstos espacios (Dictamen de la Dirección del Trabajo, 041/005, de 10 de enero de 2007; LizAMA PorTal, Luis; Ugarte Cataldo, José Luis, op. cit. (n. 2), pp. 21 - 22). Sea o no requisito de existencia de la subcontratación el elemento locativo, lo cierto es que necesariamente debe concurrir el requisito del mismo lugar de trabajo para que nazcan a la vida del derecho las obligaciones sobre prevención de riesgos laborales de la empresa principal.

- La profesora Prado señala "no puede desconocerse que el criterio del lugar será uno de los más definitorios en la aplicación práctica de las disposiciones de la ley especialmente en materia de accidentes de trabajo y lesiones a los derechos fundamentales" 
La expresión "lugar de trabajo" fue tomada del artículo $3^{10}$ del Convenio 155 de la OITsobre seguridad y salud de los trabajadores y medio ambiente de trabajo ${ }^{11}$, habiendo sido entendida para estos efectos en sentido amplio, como área o lugar determinado, edificado o no ${ }^{12}$ de la empresa principal en que se desempeñan los trabajadores contratistas y subcontratistas. De esta forma, "no debe entenderse limitado necesariamente a una unidad material específica sino que, más bien, a la consideración de si las actividades específicas de que se trata, se encuentran o no comprendidas dentro del negocio de la empresa principal"13. Se trata de cualquier lugar de trabajo al que se extiende el control directo o indirecto del empresario principal, atendiendo a la naturaleza de la actividad que desarrolla, que comprende el trabajo al aire libre, el trabajo móvil, el trabajo en alta mar, entre otros ${ }^{14}$.

Finalmente, existe un tercer requisito para el surgimiento de las obligaciones sobre de prevención de riesgos laborales de la empresa principal, que es discutible, tanto en cuando a su existencia como en cuanto a su alcance. En efecto, de acuerdo al artículo 66 bis de la Ley $\mathrm{N}^{\circ} 16.744$ -precepto legal al que se remite el artículo 183 E del Código del Trabajo- las contratas o subcontratas es necesario que sean propias del giro de la empresa principal. Exigencia que plantea dudas en cuanto a su ámbito de aplicación, surgiendo la legítima duda de si este requisito es necesario para el nacimiento de toda obligación de protección de la empresa principal respecto de los trabajadores contratistas y subcontratistas o, por el contrario, solamente es necesario para la existencia de las obligaciones previstas en el mismo artículo 66 bis de la Ley $\mathrm{N}^{\circ} 16.744$. Existiendo posiciones contradictorias al respecto. Discusión que no es irrelevante, por las consecuencias jurídicas que derivan de la respuesta que se otorgue. Para intentar solucionar este problema se recurrirá a la doctrina y jurisprudencia nacional.

El segundo problema que plantea este último requisito dice relación con su significado, no quedando claro qué significa que las contratas y subcontratas sean propias del giro de la empresa principal, existiendo dos interpretaciones posibles: una más restrictiva, que podemos llamar del "ciclo productivo" y otra, más extensiva, que podemos denominar de "las actividades indispensables" ${ }^{\prime 1}$. Para intentar clarificar su alcance se recurrirá a la doctrina española, donde existe similar discusión, a la normativa reglamentaria, cuyo alcance es discutible, y a la jurisprudencia nacional, tanto judicial como administrativa, que es escasa, pero existe, siendo muy ilustrativa. Discusión que, tampoco, es intrascendente, porque si una contrata o subcontrata no es propia del giro de la empresa principal, esta última empresa no debe cumplir las obligaciones sobre prevención de riesgos laborales establecidas en el artículo 66 bis de la Ley $\mathrm{N}^{\circ} 16.744 \mathrm{y}$, de seguirse una postura extensiva en cuanto a su campo de aplicación, tampoco con la obligación de

Prado López, Pamela. La subcontratación y el suministro en el derecho civil. Santiago, Chile: LegalPublishing, 2009, p. 26.

10 Dispone en lo pertinente: "A los efectos del presente Convenio: [...] (c) la expresión lugar de trabajo abarca todos los sitios donde los trabajadores deben permanecer o donde tienen que acudir por razón de su trabajo, y que se hallan bajo el control directo o indirecto del empleador".

1 Convenio que no ha sido ratificado por el Estado de Chile, no formando actualmente parte integrante de nuestro Ordenamiento Jurídico.

12 Artículo 4 del Decreto Supremo 76 del Ministerio del Trabajo y Previsión Social.

13 Dictamen de la Superintendencia de Seguridad Social $N^{\circ} 27.756$, de 30 de abril de 2007. <En línea $>$ [Citado 10 de noviembre de 2014] <http://www.sigweb.cl/biblioteca/DictamenesSUSESO.pdf>

14 NúÑez González, Cayetano, op. cit. (n. 2), p. 163.

15 Terminología empleada por COS para plantear la discusión existente sobre el punto en la jurisprudencia española. Véase: Cos EGEA, Manrique. "La responsabilidad solidaria de la empresa principal en materia de prevención de riesgos laborales", en: Revista Laboral y de Seguridad Social, No 338, pp. 10-19. Madrid, España: Centro de Estudios Financieros, 2011. 
Raúl Fernández Toledo / Responsabilidad de la empresa principal en prevención de riesgos laborales: ámbito de aplicación y significado del requisito contratas y subcontratas propias del giro de la empresa principal

seguridad establecida en el artículo $183 \mathrm{E}$ del Código del Trabajo ${ }^{16} \mathrm{y}$, por lo mismo, no surgiría a su respecto responsabilidad civil por el accidente laboral o enfermedad profesional experimentado por algún trabajador de la empresa contratista o subcontratista, por no serle aplicable el artículo indicado y su normativa complementaria, no existiendo obligación de protección en el trabajo incumplida que le sea imputable. Por el contrario, si es propia del giro la contrata o subcontrata, surgen las obligaciones de prevención de riesgo laborales establecidas en el artículo 66 bis de la Ley $\mathrm{N}^{\circ} 16.744^{17} \mathrm{y}$, según un sector de la doctrina, también la obligación del artículo $183 \mathrm{E}$ del Código del Trabajo, como también las responsabilidades derivadas de su incumplimiento. De ahí, la importancia de la determinación del correcto sentido y alcance del requisito establecido en el artículo 66 bis inciso $1^{\circ}$ de la Ley $\mathrm{N}^{\circ} 16.744$.

Ahora bien, independiente de la posición que se adopte sobre el requisito de las contratas y subcontratas propia del giro de la empresa principal, es claro que las obligaciones sobre prevención de riesgos laborales de la empresa principal son de hacer, teniendo una responsabilidad directa en su cumplimiento. En eso no existe discusión tanto en la doctrina ${ }^{18}$ como enla jurisprudencia judicial ${ }^{19}$ y administrativa ${ }^{20}$, la cual es unánime en que la responsabilidad en prevención de riesgos laborales de la empresa principal es directa, conllevando obligaciones de hacer.

\section{2. ÁMBITO DE APLICACIÓN DEL REQUISITO LABORES PROPIAS DEL GIRO DE LA EMPRESA PRINCIPAL REALIZADAS POR LOS CONTRATISTAS Y SUBCONTRATISTAS}

La primera duda que plantea esta exigencia establecida en el artículo 66 bis de la Ley $\mathrm{N}^{\circ}$ 16.744 es su ámbito de aplicación, no existiendo una opinión uniforme al respecto. Por lo mismo, existen dos posiciones contrapuestas, cuya diferencia radica en el diverso sentido y alcance que le otorga cada una de ellas a los artículos 183 E del Código del Trabajo y 66 bis de la Ley $\mathrm{N}^{\circ} 16.744$.

16 De seguirse la tesis que el artículo 183 E del Código del Trabajo impone a la empresa principal una obligación de seguridad igual a la del empleador, que establece el artículo 184 del Código del Trabajo, siendo esta la postura de un sector de la doctrina. En este sentido: NúÑ̃ez González, Cayetano, op. cit. (n. 2), pp. 172 - 173.

17 En la medida que se cumplan las demás exigencias establecidas en dicho precepto.

18 La existencia de responsabilidad directa ha sido reconocida por la doctrina. En este sentido: LANATa Fuenzalida, Ruth Gabriela. El contrato de trabajo como negocio jurídico en la legislación chilena. Tesina de maestría en Derecho Privado. Rosario, Argentina: Universidad Nacional de Rosario, 2007, pp. 246 - 250; Prado López, Pamela, op. cit. (n. 4), pp. 865 - 870; Ugarte Cataldo, José Luis. El nuevo derecho del trabajo. Santiago, Chile: LegalPublishing, 2008, p. 101; Diez Schwerter, José Luis, op. cit. (n. 4), p. 172; Barrientos Zamorano, Marcelo. "Sentencia de unificación sobre responsabilidad de la empresa principal en accidentes del trabajo subcontratado", en: Revista Chilena de Derecho, Vol. 40, No3, pp. 1007-1008 Santiago, Chile: Facultad de Derecho, Pontificia Universidad Católica de Chile, 2013.

19 En este sentido las sentencias de la Corte Suprema dictadas en recursos de unificación de jurisprudencia. Véase; sentencia de la Corte Suprema, 24 de abril de 2013, Rol No 5.620 - 2012; sentencia de la Corte Suprema, 25 de febrero de 2014, Rol N 9.858 - 2013; sentencia de la Corte Suprema, 10 de junio de 2014, Rol N 10.139 - 2013. <En línea> [Citada 9 de noviembre de 2014] <http://suprema.poderjudicial.cl/SITSUPPORWEB/InicioAplicacion.do.>

20 Dictamen $\mathrm{N}^{\circ} 141 / 05$ de la Dirección del Trabajo, de 10 de enero de 2007. <En línea> [Citada el 10 de diciembre de 2014] <http://www.dt.gob.d//legislacion/1611/w3-article-62810.html> 
Un sector de la doctrina ${ }^{21}$ y jurisprudencia ${ }^{22}$ sostiene que el requisito de la obra, faena o servicio objeto de la contrata y subcontrata sea propia del giro de la empresa principal es un requisito de existencia necesario para el nacimiento de todas las obligaciones sobre prevención de riesgos laborales de la empresa principal previstas en el Ordenamiento Jurídico nacional y, no solamente de las contempladas en el artículo 66 bis de la Ley $\mathrm{N}^{\circ} 16.744$. Ello porque el artículo $183 \mathrm{E}$ del Código del Trabajo consagra la obligación general de protección de la empresa principal respecto de los trabajadores contratistas y subcontratistas de conformidad con lo establecido al artículo 66 bis de la Ley $N^{\circ} 16.744$, supeditando, por tanto, su existencia a lo dispuesto en este último precepto legal ${ }^{23}$, el cual, precisamente, exige que la contratas y subcontratas sean propias del giro de la empresa principal.

De acuerdo a esta primera posición, si las contratas y subcontratas no son propias del giro de la empresa principal no surge para esta última empresa ninguna de las obligaciones de prevención de riesgos laborales reguladas en el ordenamiento jurídico nacional para la empresa principal y, por lo mismo, tampoco se les puede condenar por un tribunal de justicia civilmente a indemnizar los perjuicios sufridos por los trabajadores contratistas y subcontratistas derivados de accidentes del trabajo y enfermedades profesionales, al no haber incumplido ninguna obligación preventiva que le sea imputable, ${ }^{24}$ salvo que en virtud de un contrato, como el de prestación de servicios, se hubiere comprometido a cumplir con alguna obligación de prevención de riesgos laborales. Pacto que a la luz de la normativa laboral es totalmente lícito y, por lo mismo, eficaz, al ser favorable a los trabajadores contratistas y subcontratistas.

Otro sector, tanto de la doctrina ${ }^{25}$ como de la jurisprudencia judicial ${ }^{26}$ y administrativa ${ }^{27}$ nacional, parecen pronunciarse en contrario, en el sentido que la exigencia del giro propio sola-

21 NúÑez González, Cayetano, op. cit. (n. 2), pp. 172 - 173. En el mismo sentido parecen pronunciarse Ortiz Zavala, José Luis, Montecinos, Carolina. Ley de Subcontratación. Reglamentos de la ley de subcontratación comentados - $3^{\mathrm{a}}$ edición- Santiago, Chile: Thomson Reuters - Punto Lex, 2009, pp. 80 - 81.

22 Sentencia del Primer Juzgado de Letras del Trabajo de Santiago, 16 de agosto de 2013, RIT O - 813 - 2013. En Cfr. <http:// laboral.poderjudicial.cl/SITLAPORWEB/InicioAplicacionPortal.do> [consultada el 7 de noviembre de 2014]. Esta sentencia sostiene en lo pertinente "Undécimo: [...] el artículo $183 \mathrm{E}$ hace exigible la obligación del artículo 184 ya referida a los casos que contemplan los artículo 66 bis de la ley № 16.744 y el artículo $3^{\circ}$ del Decreto Supremo № 594, de 1999, del Ministerio de Salud. La primera disposición citada exige para los empleadores que contraten o subcontraten con otros la realización de una obra, faena o servicios propios de su giro, la obligación de velar por el cumplimiento de la normativa de higiene y seguridad. Es decir, para que la responsabilidad del artículo $183 \mathrm{~B}$ alcance al empleador principal o mandante de la obra, es necesario que éste tenga el mismo giro de la empresa contratada". También, en similares términos sentencia de la Corte de Apelaciones de Talca, 5 de noviembre de 2008, Rol No 185 - 2008. En Cfr. <http://corte.poderjudicial.cl/SITCORTEPORWEB/> [consultada el 2 de diciembre de 2014].

23 NúÑez González, Cayetano, op. cit. (n. 2), pp. 172 - 173.

24 Sentencia del Primer Juzgado de Letras del Trabajo de Santiago, 16 de agosto de 2013, RIT O - 813 - 2013, cit. (n. 22).

25 Parecen pronunciarse en este sentido Lizama Portal, Luis; Ugarte Cataldo, José Luis, op. cit. (n. 2), pp. 50 - 52; Albornoz Serrano, Marcelo; Alviz Riffo, Christian y Pérez Mendoza, Enrique. Subcontratación laboral y servicios transitorios $-3^{\mathrm{a}}$ edición-. Santiago, Chile: LegalPublishing, 2008, pp. 151 - 153.

26 Sentencia del Juzgado de Letras, Garantía y Familia de Pichilemu dictada el 10 de marzo de 2014 en causa RIT I - 21 2013, que extiende la obligación de seguridad a toda empresa principal inmersa en un trabajo en régimen de subcontratación, al establecer en el considerando sexto: " [...]no puede tomarse como equivalente el cumplimiento formal de lo mandatado en el artículo 66 bis de la ley 16.744, con el cumplimiento eficaz requerido en el artículo 183 E del Código del Trabajo, toda vez que el contenido de esta última norma es mucho más amplio y establece una obligación genérica, y directa, para las empresas principales, en orden a supervigilar eficazmente el cumplimiento de la normativa de higiene y seguridad, para de esta forma asegurar la protección de la vida y salud de los trabajadores, sea con una vinculación directa a la empresa, o subcontratados para alguna obra o faena en específico".

27 En este sentido Dictamen de la Dirección del Trabajo N².468/053, de 9 de julio de 2007. En Cfr. http://www.dt.gob.cl/ legislacion/1611/w3-article-62810.html[Consultada el 10 de diciembre de 2014]; Dictamen de la Superintendencia de Seguridad 
Raúl Fernández Toledo / Responsabilidad de la empresa principal en prevención de riesgos laborales: ámbito de aplicación y significado del requisito contratas y subcontratas propias del giro de la empresa principal

mente es condición indispensable para el nacimiento de las obligaciones previstas en el artículo 66 bis de la Ley $\mathrm{N}^{\circ} 16.744$, más no para la existencia de las obligaciones previstas en el artículo $3^{\circ}$ del Decreto 594 del Ministerio de Salud de 1999 y en el artículo 183 E del Código del Trabajo, las cuales solamente exigen que los trabajadores contratistas y subcontratistas se desempeñen en un lugar de trabajo de la empresa principal. Esto porque el artículo $183 \mathrm{E}$ del Código del Trabajo únicamente se remite al artículo 66 bis de la Ley $N^{\circ} 16.744$ para el efecto de las obligaciones previstas en ese precepto legal, más no para supeditar el nacimiento de la obligación de seguridad que consagra $y$ de las obligaciones previstas en el Decreto Supremo $N^{\circ} 594$ del Ministerio de Salud al requisito del giro propio de la contrata y subcontrata. Conclusión que reforzaría la historia fidedigna de la ley, debido a que la obligación de la empresa principal prevista en el artículo $3^{\circ}$ del Decreto Supremo $\mathrm{N}^{\circ} 594$ del Ministerio de Salud existía desde antes que la Ley $\mathrm{N}^{\circ} 20.123$ incorporara el artículo 66 bis a la Ley $\mathrm{N}^{\circ} 16.744$ y el artículo $183 \mathrm{E}$ al Código del Trabajo ${ }^{28}$, no exigiendo para su nacimiento que las contratas y subcontratas tuvieran por objeto actividades propias del giro de la empresa principal, lo que no fue modificado con la incorporación del artículo 66 bis de la Ley $\mathrm{N}^{\circ} 16.744$, cuyo propósito fue agregar obligaciones preventivas de riesgos laborales al empresario principal, adicionales a las que ya existían, más no agregar requisitos adicionales a las existentes ${ }^{29}$.

Social No 27.756 de 30 de abril de 2007; dictamen de la Superintendencia de la Seguridad Social $N^{\circ} 61.446$ de 25 de septiembre de 2007. En Cfr. http://www.sigweb.cl/biblioteca/DictamenesSUSESO.pdffconsultada el 10 de noviembre de 2014].

28 La Corte Suprema ha resuelto en dos recursos de unificación de jurisprudencia, al referirse al artículo 183 E del Código del Trabajo: "Cuarto: [...] se establece en él una obligación particular y especial para el dueño de la obra en materia de higiene y seguridad, imponiéndole el deber de protección eficaz de la vida y salud de todos los trabajadores que se desempeñen en su empresa o faena, con arreglo a las normas que en la misma disposición se expresan, esto es, el artículo 66 bis de la ley $N^{\circ} 16.744$ y artículo 3 del Decreto Supremo $N^{\circ} 594$, de 1999, del Ministerio de Salud, disposición esta última que con anterioridad a la Ley $N^{\circ} 20.123$ ya contenia la obligación de cuidado de cargo de la empresa principal" (cursiva es nuestra). Véase sentencia de 24 de abril de 2013 , pronunciada en recurso de unificación de jurisprudencia, Rol N 5.620 - 2012 y sentencia de 25 de febrero de 2014, pronunciada en recurso de unificación de jurisprudencia, Rol N$^{\circ} 9.858-2013$.

29 El Proyecto presentado por el Ejecutivo, que originó la Ley $N^{\circ} 20.123$, disponía "El dueño de la obra, empresa o faena deberá adoptar las medidas necesarias para garantizar en sus faenas la protección a los trabajadores en régimen de subcontratación en conformidad a lo establecido en el Título I del Libro II del presente Código. Sin perjuicio de las responsabilidades de la empresa contratista, el dueño de la obra, empresa o faena podrá ser fiscalizado en relación con dicha protección y sancionado si no la garantiza adecuadamente".

El Profesor Patricio Novoa señaló al respecto "el precepto en análisis sistematiza y clarifica la normativa vigente, elevándola a rango legal, pues el decreto supremo $N^{\circ}$ 594, de Salud, del 15 de septiembre de 1999, publicado en el Diario Oficial del 29 de abril de 2000, que aprobó el Reglamento sobre condiciones sanitarias y ambientales básicas en los lugares de trabajo, prescribe, en su artículo $3^{\circ}$, que la empresa está obligada a mantener en los lugares de trabajo las condiciones sanitarias y ambientales necesarias para proteger la vida y la salud de los trabajadores que en ellos se desempeñan, sean éstos dependientes directos suyos o lo sean de terceros contratistas que realizan actividades para ella", Historia de la Ley $\mathrm{N}^{\circ} 20.123$, Primer Informe Comisión Trabajo, p. 76. En Cfr.:http://www.leychile.cl/Consulta/portada_hl?tipo_norma=XX1\&nro_ley=20123 [consultada el 20 de abril de 2014]. Queda más claro que la obligación de seguridad de la empresa principal no exige que la subcontratación sea propia de su giro cuando el $183 \mathrm{E}$ del Código del Trabajo se votó en la Comisión del Senado, aprobándose por tres votos contra 2, fundando el Senador Allamand su voto de rechazo en que con dicha norma "se vuelve a las redacciones defectuosas. La lógica de la normativa debiera ser que la responsabilidad por la seguridad en el trabajo esté radicada en la empresa especializada que, precisamente, es subcontratada por esa circunstancia, pues ella es la que realmente conocerá las disposiciones aplicables en este ámbito. La empresa que la contrata no está en condiciones de conocer a cabalidad dicha normativa de seguridad. Distinto sería si se subcontratan actividades relacionadas con el giro principal'. Historia de la Ley $\mathrm{N}^{\circ} 20.123$, Informe Comisión 'Trabajo, pp. 545 - 546. En Cfr.:http://www.leychile.cl/Consulta/portada_hl?tipo_norma=XX1\&nro_ley=20123 [consultada el $20 \mathrm{de}$ abril de 2014]. La opinión del Senador Allamand deja en evidencia que con la aprobación del texto del artículo $183 \mathrm{E}$ del Código del Trabajo en la Comisión de Trabajo y Previsión Social del Senado, la obligación de seguridad surge para todas las empresas principales cuyas contratas y subcontratas se ejecutan en un lugar de trabajo que está bajo su responsabilidad, independiente del giro de las empresas, de lo contrario no habría vertido la opinión expuesta al rechazar su aprobación. 
Las consecuencias que derivan de esta segunda postura es provocar una restricción en el ámbito de aplicación del requisito de las contratas y subcontratas propias del giro de la empresa principal, debido a que solamente constituye, de acuerdo a la misma, un requisito de existencia para el nacimiento a la vida del derecho de las obligaciones preventivas (obligaciones de vigilancia y de coordinación) previstas en el artículo 66 bis de la Ley $N^{\circ} 16.744$ y en el Decreto Supremo $\mathrm{N}^{\circ}$ 76 del Ministerio del Trabajo y Previsión Social, que desarrolla el artículo 66 bis mencionado. Por el contrario, ninguna trascendencia tiene en la obligación del artículo 3 del Decreto Supremo $\mathrm{N}^{\circ}$ 594 del Ministerio de Salud y en la obligación de seguridad del artículo 183 E del Código del Trabajo $^{30}$, las cuales solamente exigen la ejecución de una contrata y/o subcontratación en un lugar de trabajo de la empresa principal. Lo que tiene incidencia directa en la responsabilidad civil, porque de acuerdo a esta segunda posición, la empresa principal puede ser responsable civilmente por siniestros laborales experimentados por trabajadores contratistas y subcontratistas en un lugar de trabajo que controle, en la medida que se acredite el incumplimiento de la obligación del artículo 183 E del Código del Trabajo y artículo 3 del Decreto Supremo $N^{\circ} 594$ del Ministerio de Salud y concurran los demás requisitos de la responsabilidad civil por accidentes del trabajo y enfermedades profesionales.

\section{DETERMINACIÓN DEL SIGNIFICADO DEL REQUISITO LABORES PROPIAS DEL GIRO DE LA EMPRESA PRINCIPAL REALIZADA POR LA CONTRATISTA Y SUBCONTRATISTA}

Asimismo, no es pacífico el significado del requisito labores propias del giro de la empresa principal establecido en el artículo 66 bis inciso $1^{\circ}$ de la Ley $\mathrm{N}^{\circ} 16.744$, necesario para imponer a la empresa principal las obligaciones previstas en dicho precepto, y según un sector de la doctrina nacional, también las reguladas en los artículos 183 E del Código del Trabajo y 3 del Decreto Supremo 594 del Ministerio de Salud.

Una alternativa es sostener que las contratas y subcontratas propias del giro equivalen al mismo giro de la empresa principal o bien que tengan conexión directa con el mismo. Ello supone que, para que puedan ser aplicados los artículos $183 \mathrm{E}$ del Código del Trabajo (según un sector de la doctrina) y 66 bis de la Ley $\mathrm{N}^{\circ} 16.744$, debe ser objeto de la contrata y/o subcontrata el núcleo esencial o fundamental de la actividad que habitualmente desarrolla la empresa principal, descartándose que pueda ser considerado como atinente al propio giro cualquier cometido accesorio o complementario impuesto por los contratantes al celebrar el acuerdo que da origen a la subcontratación ${ }^{31}$. Se trata del criterio que puede denominarse "ciclo productivo", de acuerdo al cual el círculo del propio giro de una empresa queda delimitado por las operaciones o labores que son inherentes a la producción de los bienes y servicios específicos que se propone colocar en el mercado o prestar al público ${ }^{32}$. Las actividades propias del giro, están constituidas por las operaciones necesarias para obtener el resultado con el que se identifica la empresa, debiendo existir una estrecha conexión funcional.

\footnotetext{
30 Véase el desarrollo sobre la discusión del alcance de las obligaciones sobre prevención de riesgos laborales de la empresa principal previstas en el artículo 183 E del Código del Trabajo: NúñEZ GonZÁleZ, Cayetano, op. cit. (n. 2), pp. 170 - 173. Igualmente, véase: Gajardo Harboe, María Cristina, op. cit. (n. 3), pp. 82 - 90.

31 Goerlich Peset, José María. “Coordinación de actividades empresariales y prevención de riesgos laborales”, en: Actualidad Laboral (I), pp. 142 - 143. Madrid, España: La Ley, 1997; Lizama Portal, Luis; Ugarte Cataldo, José Luis, op. cit. (n. 2 ), p. 51.

32 Cos Egea, Manrique, op. cit. (n. 15), pp. $10-11$.
} 
El supuesto más evidente es aquel en que los trabajadores contratistas y subcontratistas desarrollan las mismas labores que los trabajadores de la empresa principal, por ejecutar idéntico giro las empresas, radicando la diferencia en que la empresa principal ejecuta una parte de su actividad con sus propios trabajadores y la otra mediante empresas externas a través de la subcontratación. No externaliza una etapa del proceso productivo, sino todo el proceso productivo, pero solamente de una parte de su actividad. También existe, de acuerdo a este criterio, actividades del propio giro, cuando las distintas empresas ejecutan actividades que integran el ciclo productivo de la empresa principal. Por ejemplo, si la empresa principal es una constructora, el movimiento de tierra, la instalación de las ventanas, de la electricidad, de los techos y, en general, todas las actividades inherentes para concluir la edificación forman parte del propio giro. Lo mismo ocurre, si la empresa principal instala fachadas de vidrio, el transporte de los mismos es propio del giro; como lo estambién el montar el cableado para una compañía telefónica o de electricidad, entre otros varios ejemplos.

También serían propias del giro, las actividades que si bien no son inherentes son indispensables y permanentes para el giro de la empresa principal, como lo sería un comedor en un colegio, un bar en una discoteca, la limpieza en un hotel o restaurante. El criterio delimitador en este supuesto está constituido por la circunstancia de si la contrata o subcontrata es de producción o consumo ${ }^{33}$, debido a que solo la primera podría ser del propio giro de la empresa principal. Así, una contrata de limpieza en un hotel es de producción y propia actividad, pero no en una empresa minera, donde es de consumo y no se integra como tal. Igualmente, una contrata de construcción de una sucursal encargada por una empresa constructora es de producción y propia actividad, pero no lo es en un banco o caja de compensación, donde es de consumo, no formando de ninguna manera parte de su giro.

El concepto antes indicado es el sostenido, en nuestra opinión, por la Superintendencia de Seguridad Social, para quien las actividades propias del giro de la empresa principal son las obras o faenas que se ejecutan bajo su responsabilidad y, por ende, sujeta a su dependencia organizacional y su control ${ }^{34}$.En concepto de la Superintendencia de Seguridad Social, el criterio para definir las labores del propio giro está dado por un aspecto de dependencia organizacional, en virtud del cual la empresa principal queda excluida de las obligaciones que impone el artículo 66 bis de la Ley $\mathrm{N}^{\circ} 16.744$, en la medida que carezca de facultades para organizar y dirigir la faena ${ }^{35}$, lo que ocurre con las actividades distintas a las propias del giro de la empresa principal.

Siguiendo a la Superintendencia de Seguridad Social, para determinar si corresponde implementar la estructura preventiva prevista en el artículo 66 bis corresponde considerar no sólo a los trabajadores contratistas y subcontratistas que se encuentran expuestos a riesgos similares a los de los propios trabajadores de la empresa principal, sino también aquéllos expuestos a cualquier otro riesgo al interior de la obra o faena que se ejecuta bajo su responsabilidad y, por ende, sujeta a su dependencia organizacional y su control ${ }^{36}$. Así por ejemplo, en el caso de la construcción, es la empresa constructora y no la inmobiliaria, la responsable de implementar la estructura preventiva que establece el antes citado precepto legal, ello por cuanto la construcción de la edificación

\footnotetext{
Núñez González, Cayetano, op. cit. (n. 2), p. 163.

Dictamen $N^{\circ} 27.756$ de la Superintendencia de Seguridad Social, de 30 de abril de 2007, op cit. (n. 27).

Ibid.

Ibid.
} 
encomendada a la empresa constructora se encuentra supeditada a ésta desde el punto de vista de su ejecución y organización ${ }^{37}$ y no a la inmobiliaria, que tiene un giro distinto.

En el mismo sentido se pronuncia la escasa jurisprudencia judicial existente sobre la materia. La sentencia más clara es la recaída en un juicio sobre accidente del trabajo conocido por el Primer Juzgado de Letras del Trabajo de Santiago ${ }^{38}$, donde si bien se comprobó la existencia del trabajo en régimen de subcontratación ${ }^{39}$ y el accidente laboral, se determinó que el empleador del trabajador demandante y la empresa principal no tenían el mismo giro. Siguiendo al Tribunal de la instancia para que la responsabilidad del artículo 183 E del Código del Trabajo alcance a la empresa principal o mandante de la obra, es necesario que éste tenga el mismo giro de la empresa contratista, requisito que no concurría, puesto que la obra que se contrató era la construcción o remodelación de una sucursal de la Caja de Compensación Los Héroes, institución que se dedica al giro previsional, administrando prestaciones de dicha índole, no teniendo un giro vinculado con la construcción, que es el que desarrollaba INGEMN Ingeniería y Construcción Limitada, empleador del trabajador demandante. Conclusión que llevó al rechazo de la acción iniciada en contra de la empresa principal ${ }^{40}$. Así, entonces, no obstante haberse acreditado que el actor se desempeñó bajo régimen de subcontratación para la Caja de Compensación Los Héroes, no correspondía a esta responder de los perjuicios sufridos por el actor a consecuencia del accidente laboral, atendido a que cuenta con un giro diverso al empleador.

La sentencia antes señalada fue confirmada por la Corte de Apelaciones de Santiago, que rechazó el recurso de nulidad interpuesto por el trabajador demandante contra la sentencia definitiva dictada por el Primer Juzgado de Letras del Trabajo de Santiago ${ }^{4 !}$.

En consecuencia, si la empresa principal realiza una actividad comercial totalmente distinta a la que desarrolla la contratista, no teniendo injerencia alguna en la ejecución de la obra, faena o servicio de la subcontratación, la que se desarrolla bajo la responsabilidad de la empresa contratista, el objeto de la contrata es distinta a las labores propias del giro de la empresa principal. En otros términos, la empresa principal y la contratista tienen giro diferente, de acuerdo a la normativa nacional, cuando el objeto de la subcontratación es una obra, faena o servicio que

\footnotetext{
37 Ibid.

38 Sentencia del Primer Juzgado de Letras del Trabajo de Santiago, 16 de agosto de 2013, RIT O - 813 - 2013, op cit. (n. 22).

3. Concluye en lo pertinente: "UNDÉCIMO: [...] Que tal como se aprecia, del tenor del contrato antes mencionado, se advierte que entre las demandadas principal y solidaria hay un vínculo contractual, en virtud del cual la demandada Ingemm Ingeniería y Construcción Limitada, se obliga a ejecutar labores referidas a la remodelación de la sucursal de Los Héroes, para lo cual deberá ceñirse a las instrucciones dadas por la demandada los héroes.

Que del tenor de la vinculación antes mencionada, aparece que la demandada Ingemm Ingeniería y Construcción Limitada, está ejecutando obras o servicios por su cuenta y riesgo con trabajadores propios, para la demandada Caja de Compensación Los Héroes, quien a todas luces aparece como dueño de la obra, empresa o faena, adquiriendo así la calidad de empresa principal en un régimen de subcontratación. De esta manera, atendido a que el trabajador se desempenaba en dependencias de la sucursal de Los Héroes en la comuna de Catemu, trabajando para la demandada Ingemm Ingeniería y Construcción Limitada, a juicio de este sentenciador el actor se encontraba bajo régimen de subcontratación para la demandada Caja de Compensación Los Héroes. De esta manera, aparece que la fecha del accidente, la referida demandada tenía la calidad de empresa principal. Ellos se manifiesta claramente en la redacción de la cláusula $16^{\mathrm{a}}$ del contrato de construcción a suma alzada, en el que se establecen las obligaciones del contratista y la de la empresa mandante, todo lo cual está regulado en el artículo 183 A del Código del Trabajo".

40 Sentencia del Primer Juzgado de Letras del Trabajo de Santiago, 16 de agosto de 2013, RIT O - 813 - 2013, considerando undécimo. Vid. (n. 22).

41 Sentencia de la Corte de Apelaciones de Santiago, 9 de enero de 2014, Rol Reforma Laboral No 1.365 - 2013. En Cfr.: http:// corte.poderjudicial.cl/SITCORTEPORWEB/ [consultada el 2 de diciembre de 2014].
} 
Raúl Fernández Toledo / Responsabilidad de la empresa principal en prevención de riesgos laborales: ámbito de aplicación y significado del requisito contratas y subcontratas propias del giro de la empresa principal

no corresponde al giro propio de la empresa principal, lo que ocurrirá cuando su ejecución no se realiza bajo su responsabilidad sino de la contratista ${ }^{42}$.

El objeto de la contrata es diferente al giro de la empresa principal en cualquier situación en que el contratista ejecuta su actividad en condiciones en que la empresa principal no posee responsabilidad y control sobre ello. Ello se puede encontrar en los supuestos en que la empresa principal ha subcontratado o externalizado la totalidad de su propio giro, debido a que no tendrá la tuición ejecutiva u operativa sobre la gestión del contratista ${ }^{43}$. El mismo criterio se aplica a aquellas actividades externalizadas sin posibilidad de ejercer control sobre las mismas, como el caso de las empresas de transporte de carga que se contratan para transportar materias primas, insumo o productos terminados de la empresa principal ${ }^{44}$.

Una segunda posición sobre el alcance de las contratas o subcontratas propias del giro de la empresa principal, es la que podemos llamar de "las actividades indispensables", que dilata el alcance de aquellas a todas las labores, específicas o inespecíficas que una determinada organización productiva debe desarrollar para realizar adecuadamente sus funciones ${ }^{45}$. Este criterio asigna una fuerza expansiva a las actividades propias del giro, excluyendo la identificación entre labores del "mismo" y "propio" giro, pues con ello se haría prácticamente inaplicable los artículos $183 \mathrm{E}$ del Código del Trabajo y 66 bis de la Ley $\mathrm{N}^{\circ} 16.744$. Las labores serían del propio giro siempre que sea advertible una conexión directa o indirecta con el proceso productivo ${ }^{46}$. Para determinar si esta conexión existe se acude al criterio de la sustituibilidad de la contrata, existiendo siempre que el empresario principal pudiere conseguir el mismo resultado sin recurrir a terceros contratistas ${ }^{47}, y$ siendo difícil encontrar actividades que no sean sustituibles, el ámbito del propio giro, de acuerdo a esta posición, es razonablemente extensivo a todo cuanto una empresa realice en su carácter de $\mathrm{tal}^{48}$. Confirmaría que este es el criterio adoptado por el Ordenamiento Jurídico nacional, la definición establecida en el artículo $4^{\circ}$ del Decreto Supremo 76, conforme a la cual, se entiende "por obra, faena o servicios propios de su giro, todo proyecto, trabajo o actividad destinado a que la empresa principal desarrolle sus operaciones o negocios, cuya ejecución se realice bajo su responsabilidad, en un área o lugar determinada, edificada o no, con trabajadores sujetos a régimen de subcontratación"49. Definición que es de una amplitud tal que difícilmente podría excluir a alguna contrata o subcontrata, lo que se traduce en una gran ventaja en términos de garantías para los

42 Se ha resuelto: "Quinto: Que, como se dijo en el Considerando Tercero, el giro de la empresa reclamante es el de Exportaciones y Corretaje de productos agrícolas, actividad totalmente alejada de los trabajos de construcción, los que naturalmente deben ser realizados por una empresa dedicada a dicho rubro, como aconteció en la especie, sin que, como se señalara más arriba, tuviere Unifrutt'Traders, ingerencia alguna en su ejecución, el que se desarrolló bajo la responsabilidad de la empresa contratista, Constructora Lyon y Cía. Ltda.

De tal modo no puede considerarse que la faena de pintura en altura en el packing ubicado en Teno, corresponda al giro propio de la empresa principal, en los términos establecidos en el artículo 4 del Decreto 76 referido en el Fundamento Primero, desde el momento que no se realizaron bajo su responsabilidad sino de la contratista" (sentencia de la Corte de Apelaciones de Talca, 5 de noviembre de 2008, Rol N 185 - 2008. Vid. [n. 22]) En igual sentido Albornoz Serrano, Marcelo; AlvizRiffo, Christian; Pérez Mendoza, Enrique, op. cit. (n. 25), pp. $169-170$.

43 Albornoz Serrano, Marcelo; Alviz Riffo, Christian y Pérez Mendoza, Enrique, op. cit. (n. 25), p. 170.

44 Ibid.

45 Cos Egea, Manrique, op. cit. (n. 15), p. 11.

46 Goerlich Peset, José María, op. cit. (n. 31), p. 143.

47 Ibid.

48 Ibid.

49 Núñez GonzÁlez, Cayetano, op. cit. (n. 2), p. 163. 
trabajadores contratistas y subcontratistas que trabajan en el espacio productivo controlado por la empresa principal ${ }^{50}$.

Conforme a esta segunda posición, difícilmente una contrata o subcontrata quedaría excluida del ámbito de protección y responsabilidad de la empresa principal, por no decir que no existiría contrata ni subcontrata respecto de la cual no fuere aplicable el artículo 66 bis de la Ley $\mathrm{N}^{\circ}$ 16.744 y el artículo 183 E del Código del Trabajo junto a sus normas complementarias, si se estima que la exigencia del giro propio es una requisito necesario para la existencia de las obligaciones preventivas reguladas en las disposiciones señaladas.

\section{CONCLUSIONES}

La normativa nacional extiende la obligación general de proteccióndel empleador regulada en el artículo 184 del Código del Trabajo a la empresa principal respecto de los trabajadores de los contratistas y subcontratistas. También, consagra obligaciones de vigilancia y coordinación en la prevención de riesgos laborales para la empresa principal (artículo 66 bis de la Ley $N^{\circ} 16.744$ ). De este modo, la legislación nacional aborda la regulación de la subcontratación de obras y servicios desde una doble perspectiva, prevencionista y represora.

Así, con las obligaciones de seguridad, vigilancia y coordinación impuestas al empresario principal se pretende, desde una óptica prevencionista que este no se desentienda de los trabajadores de los contratistas y subcontratistas sino que, por el contrario, se involucre en la protección de su seguridad y salud mediante una actuación directa y fiscalizadora de las empresas empleadoras de dichos trabajadores.

Como mecanismo de garantía del cumplimiento de estos deberes preventivos la normativa contempla sanciones para la empresa principal frente a la inobservancia de las obligaciones preventivas previstas en el Ordenamiento Jurídico. Y a la vez, una responsabilidad civil derivada de los accidentes del trabajo y enfermedades profesionales que sufran los trabajadores contratistas y subcontratistas, como consecuencia del incumplimiento de sus obligaciones preventivas.

Se observa que es discutido el ámbito de aplicación del requisito de las contratas y subcontratas propias del giro de la empresa principal, pronunciándonos a favor del criterio que sostiene que solamente es una exigencia para la procedencia de las obligaciones de protección establecidas en el artículo 66 bis de la Ley $\mathrm{N}^{\circ} 16.744$ (vigilancia y coordinación), más no para el nacimiento de la obligación de seguridad regulada en el artículo 183 E del Código del Trabajo (si es admite que existe) y la del artículo 3 del Decreto Supremo $N^{\circ} 594$ del Ministerio de Salud. Interpretación que, por lo demás, resulta más ajustada al principio del Derecho del Trabajo in dubio pro operario, conforme al cual frente o dos o más interpretaciones posibles de una norma laboral debe preferirse la más favorable al trabajador ${ }^{51}$.

También resulta problemático el sentido del requisito indicado, en cuanto a su significado, defendiendo la teoría del ciclo productivo. Esto porque si bien, resulta de trascendental importancia la responsabilidad de la empresa principal en prevención de riesgos laborales respecto de los trabajadores contratistas y subcontratistas, no es razonable efectuar una interpretación injustificadamente extensiva de los requisitos necesarios para el nacimiento de dicha responsabilidad. De

\footnotetext{
50 Ibid.

51 Plá Rónríguez, Américo. Los Principios del Derecho del Trabajo. - $3^{a}$ edición actualizada-. Buenos Aires, Argentina: Ediciones Depalma, 1998, pp. 84 - 87.
} 
Raúl Fernández Toledo / Responsabilidad de la empresa principal en prevención de riesgos laborales: ámbito de aplicación y significado del requisito contratas y subcontratas propias del giro de la empresa principal

ahí, que el requisito que las contratas y subcontratas sean propias del giro de la empresa principal, se debe determinar conciliando los intereses de los trabajadores y la empresa principal, siendo exigible a esta última las obligaciones preventivas en la medida que se encuentre en posición de cumplirlas razonablemente considerando no sólo a los trabajadores que se encuentran expuestos a riesgos similares a los de sus propios trabajadores, sino que a los trabajadores en general, respecto de cualquier otro riesgo que pudiera afectarlos al interior de la obra o faena que se ejecuta bajo su responsabilidad y, por ende, sujeta a su dependencia organizacional y su control. Por tanto, el criterio para determinar la responsabilidad preventiva de la empresa principal respecto de las obligaciones preventivas establecidas en el artículo 66 bis de la Ley $\mathrm{N}^{\circ} 16.744$ es que las labores descentralizadas se encuentren bajo su dependencia organizacional y su control, puesto que ello le permite estar en mejores condiciones para conocer los riesgos a que se hallan expuestos los distintos trabajadores y, por lo mismo, puede de mejor forma adoptar las medidas preventivas. No ocurre lo mismo, con las obras y servicios que escapan a su control, de las que no está en condiciones de conocer los riesgos que pueden afectar a los trabajadores contratistas y subcontratistas, no pudiendo de modo eficaz adoptar las medidas preventivas, al desconocer los riesgos. Lo que ocurre con las actividades que no tienen conexión con el giro que explota, las que no serán propias de su giro, no siéndole exigible las obligaciones contempladas en el artículo 66 bis de la Ley $\mathrm{N}^{\circ}$ 16.744.

Es por ello que se defiende el criterio del ciclo productivo, al ser el que más se ajusta a la normativa vigente y a los intereses de las partes involucradas, no siendo muy restrictivo ni muy extensivo, descartándose el criterio de los servicios indispensables. Este último criterio extiende en demasía el ámbito de aplicación del artículo 66 bis de la Ley $\mathrm{N}^{\circ} 16.744$, haciéndolos aplicables, en general, a todas las contratas y subcontratas que se ejecutan en el lugar de trabajo de la empresa principal, haciendo caso omiso que el requisito de labores del propio giro implica que el legislador está pensando en una extensión razonable de la responsabilidad preventiva de la empresa principal, no ampliándola indiscriminadamente. Pues, de haber sido ello así el legislador no habría establecido el requisito que las contratas y subcontratas fueran del propio giro de la empresa principal.

Por lo demás, para determinar el ámbito de aplicación del artículo 66 bis de la Ley $\mathrm{N}^{\circ}$ 16.744 no corresponde considerar, en nuestra opinión, la definición que otorga el artículo $4^{\circ}$ del Decreto Supremo 76 , toda vez que esta normativa reglamentaria no tiene por función definir la expresión "contratas y subcontratas de obras, faenas o servicios propios del giro de la empresa principal" que contempla la disposición legal indicada en su inciso $1^{\circ}$. Su función es determinar los requisitos necesarios para la constitución y funcionamiento de los Comités Paritarios de Higiene y Seguridad y Departamentos de Prevención de Riesgos de obras, faenas o servicios propios del giro de la empresa principal. El tenor literal del inciso final del artículo 66 bis es claro en cuanto al ámbito de aplicación del Decreto Supremo $76^{52}$ no admitiendo dudas de su alcance. Cuestión distinta es que el Decreto Supremo haya excedido el mandato legal, caso en el cual estaría en duda su validez, puesto que la normativa reglamentaria no puede exceder ni contradecir la disposición que permite su dictación, al ser de rango jerárquico superior y su fuente de legitimidad.

52 Señala el inciso final: “Asimismo, corresponderá al mandante, velar por la constitución y funcionamiento de un Comité Paritario de Higiene y Seguridad y un Departamento de Prevención de Riesgos para tales faenas, aplicándose a su respecto para calcular el número de trabajadores exigidos por los incisos primero y cuarto, del artículo 66, respectivamente, la totalidad de los trabajadores que prestan servicios en un mismo lugar de trabajo, cualquiera sea su dependencia. Los requisitos para la constitución y funcionamiento de los mismos serán determinados por el reglamento que dictará el Ministerio del Trabajo y Previsión Social". 
El alcance de la expresión "contratas y subcontratas de obras, faenas o servicios propios del giro de la empresa principal" debe determinarse acudiendo a las reglas de interpretación de la ley establecidas en los artículos 19 al 24 del Código Civil, que son de general aplicación. Y aun, de sostenerse que corresponde aplicar la definición contenida en el artículo 4 del Decreto Supremo 76, el énfasis debe colocarse en que la ejecución de las obras, faenas o servicios que la empresa principal contrate y subcontrate a otros "se realicen bajo su responsabilidad", que es precisamente el criterio adoptado por la Superintendencia de Seguridad Social cuando se ha pronunciado sobre el alcance de dicho artículo $4^{\circ}$, no extendiendo la estructura preventiva del artículo 66 bis a toda contrata y subcontrata, sino solo a aquellas que puede controlar y dirigir bajo su responsabilidad. Criterio que se ajusta más a la posición del ciclo productivo que a la postura de las actividades indispensables.

No se puede aplicar injustificadamente el requisito analizado. Extender la aplicación del concepto "obras, faenas o servicios propios de su giro", a actividades absolutamente ajenas al giro de la empresa principal y ejecutadas bajo responsabilidad de contratistas especializados, llevaría al absurdo de suponer que en todo trabajo o faena que se encargue a terceros haría surgir las obligaciones preventivas previstas por el artículo 66 bis de la Ley $\mathrm{N}^{\circ} 16.744$ para la empresa principal, pues de una forma $u$ otra, sea directa o indirectamente, todo trabajo iría en beneficio de aquella ${ }^{53}$. Mal se puede exigir a la empresa principal que cumpla las obligaciones de vigilancia y coordinación en la prevención de riesgos laborales a que se hallan expuestos los trabajadores contratistas y subcontratistas que se desempeñan en faenas que no son de su giro, que no las controlan ni supervisan y que se desarrollan de manera temporal en sus instalaciones, lo que ocurriría con la interpretación extensiva de la exigencia señalada.

Corresponderá, en definitiva, a los órganos del Estados competente, estos es, a los Tribunales de Justicia, a la Superintendencia de Seguridad Social, a la Inspección del Trabajo y a los Servicios de Salud (SEREMI), dentro de su ámbito de competencia, determinar el ámbito de aplicación del requisito que las contratas y subcontratas sean propias del giro de la empresa principal como su correcto sentido, para establecer si la empresa principal debe cumplir con las obligaciones preventivas que establece el ordenamiento jurídico nacional respecto de los trabajadores contratistas y subcontratistas como también la responsabilidad que le corresponde de ser sujeto deudor de la labor preventiva, en caso de incumplimiento de las obligaciones de que es sujeto pasivo.

\section{BIBLIOGRAFÍA}

Albornoz Serrano, Marcelo; Alviz Riffo, Christian; Pérez Mendoza, Enrique. Subcontratación laboral y servicios transitorios. $-3^{\mathrm{a}}$ edición-. Santiago, Chile: Legal Publishing, 2008.

Barrientos Zamorano, Marcelo. "La obligación de seguridad en la subcontratación laboral: previsibilidad del hecho y del daño", en: Revista Chilena de Derecho, Vol. 39, N 1, pp. 77 - 111. Santiago, Chile: Facultad de Derecho, Pontificia Universidad Católica de Chile, 2012,

Barrientos Zamorano, Marcelo. "Sentencia de unificación sobre responsabilidad de la empresa principal en accidentes del trabajo subcontratado", en: Revista Chilena de Derecho, Vol. 40, N 3, pp. 1001 - 1016. Santiago, Chile: Facultad de Derecho, Pontificia Universidad Católica de Chile, 2013.

Cos Egea, Manrique. "La responsabilidad solidaria de la empresa principal en materia de prevención de riesgos laborales", en: Revista Laboral y de Seguridad Social, No 338, pp. 5-30. Madrid, España: Centro Estudios Financieros, Madrid, 2011.

53 Sentencia de la Corte de Apelaciones de Talca, 5 de noviembre de 2008, Rol No 185 - 2008. Vid. (n. 22). 
Raúl Fernández Toledo / Responsabilidad de la empresa principal en prevención de riesgos laborales: ámbito de aplicación y significado del requisito contratas y subcontratas propias del giro de la empresa principal

Diez SCHWERTER, José Luis. "Responsabilidad civil derivada de accidentes del trabajo y enfermedades profesionales. Aspectos relevantes de su regulación y operatoria actual", en: Revista de Derecho, $\mathrm{N}^{\circ} \mathrm{XXXI}$, pp. 163-185.Valparaíso, Chile: Escuela de Derecho, Pontificia Universidad Católica de Valparaíso, 2008.

Diez SCHWERTER, José Luis. "Responsabilidad civil derivada de accidente de trabajo y enfermedades profesionales en Chile: evolución, funcionamiento y propuestas de racionalización (segunda parte)", en: Revista de Derecho, $\mathrm{N}^{\circ} 225-226$, pp. $239-291$. Concepción, Chile: Facultad de Derecho Universidad de Concepción 2009.

Gajardo Harboe, María Cristina. "Obligaciones en materia de higiene y seguridad en el trabajo: los cambios de la Ley de Subcontratación”, en: Revista Laboral Chilena, año 19, N 176, pp. 81-95. Santiago, Chile, 2009.

Goerlich Peset, José María. “Coordinación de actividades empresariales y prevención de riesgos laborales", en: Actualidad Laboral (I), pp. 125 - 156. Madrid, España: La Ley, 1997

Lanata Fuenzalida, Ruth Gabriela. El contrato de trabajo como negocio jurídico en la legislación chilena. Tesina de maestría en Derecho Privado (inédita). Rosario, Argentina: Universidad Nacional de Rosario, 2007.

Lizama Portal, Luis; Ugarte Cataldo, José Luis. Subcontratación y Suministro de Trabajadores. Santiago, Chile: LexisNexis, 2007.

Miñarro Yanini, María. "La prevención de riesgos laborales en las contratas y subcontratas", en: Documentación Laboral, $\mathrm{N}^{\circ} 68$ (Vol. II) pp. 83-11. Madrid, España, 2003.

NúÑez González, Cayetano. Prevención de riesgos laborales en Chile. Alcance y contenido del artículo 184 del Código del Trabajo. Santiago, Chile: Librotecnia, 2013.

Ortiz Zavala, José Luis; Montecinos, Carolina Ley de Subcontratación. Reglamentos de la ley de subcontratación comentados. - $3^{2}$ edición-. Santiago, Chile: Thomson Reuters - Punto Lex, 2009.

PalaVecino Cáceres, Claudio. Subcontratación. Régimen jurídico del trabajo subcontratado y del suministro de personal. Santiago, Chile: Editorial Jurídica, 2006.

PÉrez Mendoza, Enrique. Normas de seguridad y salud en el trabajo establecidas en la Ley $N^{\circ} 20.123$ y en el Reglamento $N^{\circ}$ 76/2007 del Ministerio del Trabajo y Previsión Social. Documento inédito sin publicar.

Plá Ródríguez, Américo. Los Principios del Derecho del Trabajo. - $3^{2}$ edición actualizada-. Buenos Aires, Argentina: Ediciones Depalma, 1998.

PRado López, Pamela. "Crítica a la configuración de la responsabilidad civil de la empresa principal y de la empresa usuaria en la Ley $\mathrm{N}^{\circ} 20.123$ ". En A.A.V.V. Estudios de Derecho Civil III. Jornadas Nacionales de Derecho Civil Valparaíso 2007. Santiago, Chile: LegalPublishing, 2008, pp. 857 - 872.

Prado López, Pamela. La subcontratación y el suministro en el derecho civil. Santiago, Chile: LegalPublishing, 2009.

Ugarte Cataldo, José Luis. El nuevo derecho del trabajo. Santiago, Chile: LegalPublishing, 2008. 